Questions vives

\section{Questions Vives}

Recherches en éducation

$N^{\circ} 25$ | 2016

L'activité des enseignants face au décrochage scolaire : Quelles difficultés et quelles mises en œuvre professionnelles?

\title{
Les TIC(E) dans une démarche de soins-études. Enquête auprès d'équipes pédagogiques et d'élèves hospitalisés
}

ICT(E) in hospital education. Survey on hospital teachers and hospitalized pupils

\section{Séverine Colinet}

\section{(e) OpenEdition \\ Journals}

\section{Édition électronique}

URL : http://journals.openedition.org/questionsvives/1965

DOI : 10.4000/questionsvives.1965

ISSN : 1775-433X

\section{Éditeur}

Université Aix-Marseille (AMU)

\section{Édition imprimée}

Date de publication : 7 juillet 2016

ISBN : 978-2-912643-49-0

ISSN : 1635-4079

Référence électronique

Séverine Colinet, « Les TIC(E) dans une démarche de soins-études. Enquête auprès d'équipes

pédagogiques et d'élèves hospitalisés », Questions Vives [En ligne], № 25 | 2016, mis en ligne le 05 septembre 2016, consulté le 19 avril 2019. URL : http://journals.openedition.org/questionsvives/1965 ; DOI : 10.4000/questionsvives. 1965

Ce document a été généré automatiquement le 19 avril 2019.

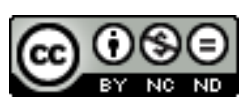

Questions Vives est mis à disposition selon les termes de la licence Creative Commons Attribution Pas d'Utilisation Commerciale - Pas de Modification 4.0 International. 


\title{
Les TIC(E) dans une démarche de soins-études. Enquête auprès d'équipes pédagogiques et d'élèves hospitalisés
}

ICT(E) in hospital education. Survey on hospital teachers and hospitalized pupils

\author{
Séverine Colinet
}

\section{Introduction}

1 Les sciences de l'éducation et la sociologie des usages ont mis en évidence les enchevêtrements et la complexité des différents types d'usages et d'utilisations des TIC (E). Ces usages dépendent à la fois des cadres techniques et sociaux. Nous avons souhaité les analyser dans un contexte spécifique peu investi par les chercheurs, celui des élèves en soins-études. L'objectif de cette recherche qualitative est de comprendre la place et les usages des TIC(E) pour les élèves qui, souffrant d'une maladie chronique et/ou connaissant une situation de handicap, utilisent les TIC(E) dans leur parcours de soinsétudes. Plus que de connaître les usages et les utilisations des TIC(E) propres aux élèves hospitalisés, nous avons voulu comprendre les rapports qu'entretiennent les professionnels - enseignants et directeurs d'établissement - qui accompagnent les élèves en soins-études dans leurs pratiques des TIC(E). Le recueil des données s'effectue sur des situations d'apprentissage instrumentées peu étudiées, ce qui constitue une originalité de cet article.

2 Après avoir dressé un état des lieux bibliographique pour contextualiser notre objet de recherche, nous présenterons la méthodologie mobilisée pour cette enquête, avant de décrire les objectifs institutionnels de l'intégration des TIC(E) mis en évidence dans la démarche de soins-études, en les croisant avec les perceptions des élèves. Par la suite sont présentés les rapports que les équipes pédagogiques entretiennent avec les TIC(E) ainsi que certains usages et utilisations qu'en font les élèves, à quoi s'ajoutent les principales 
utilisations des TIC(E) par les élèves hospitalisés avant et après leur hospitalisation, utilisations également mises en rapport avec les types de pathologies des élèves.

\section{Cadrage théorique}

\subsection{Problématique et objet de la recherche}

3 La question centrale problématisée de cet article a consisté à comprendre les types d'usages et d'utilisations des TIC(E) par les élèves hospitalisés, et à déterminer comment les professionnels les accompagnant intègrent les TIC(E) dans leur démarche. Dans une visée comparative, notre enquête a porté sur les usages et les utilisations des $\operatorname{TIC}(\mathrm{E})$ en contexte hospitalier et hors hospitalier, et nous avons cherché à mieux saisir les formes d'usages et d'utilisations entre l'avant et pendant hospitalisation des élèves. Ce travail s'inscrit à la croisée du champ des sciences de l'éducation et de la sociologie des usages.

\subsection{Définitions}

4 Notre recherche bibliographique centrée sur la notion d'usage, nous a également amenée à expliciter des notions connexes : utilisation, utilité, autonomie, autodétermination et dispositif.

5 La notion d'usage n'est pas un objet naturel mais un construit social (Chambat, 1994, p. 253) qui est abordé selon plusieurs axes: la généalogie des usages, le processus d'appropriation, l'élaboration du lien social, l'intégration des usages dans les rapports sociaux et nous ajouterons, compte-tenu du contexte de notre enquête, les rapports pédagogiques et didactiques. La perception de l'utilité influence les intentions d'utilisation (Davis, 1989), l'utilité étant définie en termes d'efficience par rapport à une tâche à accomplir. Selon Chaptal (2007), l'utilisation renvoie à la fois à une action ponctuelle et aux aspects manipulatoires quand «usages " s'entend en tant qu'usages sociaux (Baron \& Bruillard, 1996), action communément observée dans un groupe. Moeglin (2005) définit les usages comme « des utilisations inscrites dans le temps long de pratiques éducatives et sociales stabilisées » (Lacroix et al, 1993, p. 101). Les usages, quant à eux résultent des transformations que, collectivement plus souvent qu'individuellement, ils imposent aux cadres fixés par l'offre et les politiques (Moeglin, 2005, p. 160). L'usager, ici, est bien considéré comme acteur.

6 La notion d'autonomie sociale a largement irrigué les travaux de la sociologie des usages (Jouët, 2000). L'autonomie peut s'évaluer, selon la typologie de Boutet et al (2003) qui scinde l'autonomie de « décision » et celle « d'exécution ». L'autonomie est une part de la définition d'autodétermination (Vehmeyer \& Bolding, 1999, p. 355) regroupant également les notions d'autorégulation, d'empowerment et d'autoréalisation. L'autonomie exprime la capacité des êtres humains à raisonner en conscience, à faire preuve de réflexivité et à s'autodéterminer (Le Coadic, 2006).

7 L'autodétermination se définit comme « des habiletés et les attitudes requises chez une personne, lui permettant d'agir directement sur sa vie en effectuant librement des choix non influencés par des agents externes inclus » (Sands \& Wehmeyer 1996).

Quant au terme de «dispositif», avant de se référer à une réalité ontologique, il représente surtout un concept heuristique ou épistémologique (Peeters \& Charlier, 1999). 
Le dispositif peut être conçu comme un composé «d'humain » et de "non-humain » (Callon \& Latour, 1991) et d'interdépendances, l'usage de ce concept n'étant pas étranger à une technicisation grandissante des environnements quotidiens (Leblanc, 1998). Les dispositifs attendent de l'usager qu'il s'approprie les ressources mises à sa disposition.

\subsection{TIC(E) éducation et santé}

9 À partir de la définition de Baron et Bruillard (2008, p. 3), reprise notamment par Aoudé (2011, pp. 15-16) :

L'acronyme TIC serait à «l'articulation, d'une part des objets techniques (qu'ils soient des ordinateurs, téléphones... ou des applications, logiciels, plate-forme...) avec, d'autre part, les activités qu'on fait avec, et ce, qu'elles soient ou non directement prescrites (par un adulte, le travail, l'école...) ». Quant aux TICE, elles constituent «un domaine de la technologie de l'éducation consacré à la recherche et aux applications pédagogiques qui se rapportent spécifiquement aux démarches, aux approches, aux procédés et aux techniques d'enseignement-apprentissage qu'impliquent les actions pédagogiques intégrant l'usage des outils numériques » (Quintin, 2014).

\subsubsection{TIC(E) et éducation}

11 Dans le cadre de la loi d'orientation et de programmation pour la refondation de l'École (2013) et plus spécifiquement du plan numérique pour l'éducation, le Ministère de l'Éducation nationale, de l'enseignement supérieur et de la recherche a annoncé la généralisation des usages et du développement des ressources numériques dans les établissements scolaires. Au niveau de la recherche, il a été montré que les TICE, comme la formation ouverte et à distance (FOAD), permettent un enseignement plus souple car elles remettent en question la règle des trois unités de lieu, de temps et d'action de l'enseignement en classe et offrent des dispositifs plus individualisés (Bahry \& Debon, 2009), même si elles présentent des limites certaines, notamment des taux d'abandons dans les usages.

\subsubsection{Champ de la santé et $\operatorname{TIC}(E)$}

Le programme lancé relatif à l'hôpital numérique (Ministère chargé de la santé, 2013) a défini la stratégie nationale des systèmes d'information hospitaliers pour la période 2012-2017, dans un contexte national de volonté politique de scolarisation égale pour les personnes ayant un handicap (indiqué dans la loi du 11 Février 2005). L'usage des technologies de l'information et de la communication pour l'enseignement (TICE) est clairement encouragé aux plans national (Haute Autorité de Santé, 2011) et européen, y compris pour les 11000 enfants et adolescents hospitalisés chaque année.

Au niveau des recherches, l'usage des TIC dans le champ de la santé porte essentiellement sur leurs logiques d'implantation (Grosjean \& Bonneville, 2007), sur les échanges dans le cadre de forums de discussion (Akrich \& Méadel, 2002), sur l'éducation thérapeutique (Harry, Gagnayre \& d'Ivernois, 2008), sur la consommation d'informations médicales (Nabarette, 2002) ou encore sur les compétences et les usages des TIC dans le cadre de l'éducation à la santé. Les publications relatives à la scolarité dans un contexte de soins sont assez peu nombreuses. Quelques-unes portent sur le rapport à l'apprentissage pour des élèves malades ou accidentés (Bourdon \& Roy, 2006 ; Romano, 2006; Voisin, 2003). 
Lorsque l'on s'intéresse au contexte hospitalier, la recherche sur l'usage des TICE est là encore très limitée (Strutz \& Marquet, 2008). On constate que la recherche sur les usages et les utilisations des technologies relatives à un contexte scolaire hospitalier constitue plus souvent des illustrations venant appuyer des réflexions plus larges sur le rapport à la scolarité, que véritablement des enquêtes portant spécifiquement sur le contexte hospitalier. C'est pourquoi notre recherche aura pour ambition de combler le manque bibliographique relatif à l'usage des TIC(E) par des élèves connaissant un parcours scolaire hospitalier.

\section{Contexte des jeunes hospitalisés en « soins- études »}

Notre enquête s'est réalisée dans deux centres médicaux et pédagogiques adaptés (CMPA) et un Établissement Régional d'Enseignement Adapté (ÉREA). Ces établissements sont rattachés à l'Éducation Nationale. Ils présentent la spécificité de disposer d'une unité de soins-études. Le "projet éducatif et scolaire [se situe] autour d'une prise en charge globale du jeune associant les soins et les études" comme l'indique la plaquette institutionnelle d'un CMPA. Le concept de "soins-études" mobilise une équipe pluridisciplinaire des secteurs du médical, du paramédical, de l'intervention sociale et de l'enseignement.

Les classes et les niveaux sont multiples (niveaux CE2, CM1 et CM2, collège, SEGPA, CAP, Lycée général et professionnel). Le dispositif de soins-études est une phase de transition visant à favoriser le retour progressif dans le milieu scolaire ordinaire. Il requiert un projet pédagogique personnalisé pour le patient-élève et cela dès son admission. Ce dernier dispose donc d'un emploi du temps personnalisé en fonction de sa prise en charge médicale, rééducative et de son projet scolaire ou professionnel. Selon les besoins repérés, le projet pédagogique personnalisé peut prendre différentes formes: cours collectifs, ateliers en petits groupes, tutorat et travail individuel. La scolarisation peut être complète (« similaire » au milieu ordinaire), partielle (quelques disciplines en classe), prise en charge par groupe spécifique (réadaptation) ou prise en charge individuellement. En fonction de la pathologie ou de la nature du handicap, les temps et les formes d'hospitalisation et de scolarisations sont différents et plus ou moins complexes.

\section{Méthodologie}

Cet article repose sur l'analyse d'une dizaine de comptes-rendus d'observations nonparticipantes en classes et dans des temps péri- et extra-scolaires. Ont également été conduits 59 entretiens semi-directifs dont 5 entretiens-tests avec le support de guides d'entretien. Les observations et les comptes-rendus correspondant visaient à mieux saisir les usages des TICE dans les pratiques quotidiennes, les entretiens semi-directifs ayant pour objet de compléter cette approche en permettant aux différents acteurs d'expliciter leurs pratiques et de permettre plus spécifiquement aux élèves, de verbaliser les évolutions entre l'avant et l'après hospitalisation. Cette explicitation est également pour les acteurs une manière d'entrer dans une démarche réflexive. Les entrevues ont été enregistrées auprès de l'ensemble des acteurs ayant participé à l'enquête - différentes catégories de personnels et élèves - concernés par les TIC(E) dans un contexte 
simultanément hospitalier et scolaire. Nous avons donc interviewé des enseignants (25) dont 5 référents TICE, un directeur (3) et un directeur adjoint (1). Nous nous sommes également entretenue avec des élèves (30) des premier et second degrés qui suivent un cursus scolaire, au sein de l'hôpital, pour une période de 6 mois à un an et plus. L'échantillon de cette recherche qualitative est donc non représentatif et nonprobabiliste. La conduite de cette recherche a exigé l'obtention du consentement des familles à la participation de leurs enfants à l'enquête.

Lors des entretiens avec les élèves, nous avons rencontré deux obstacles d'ordre méthodologique. Tout d'abord, ces élèves rencontraient de fortes difficultés à verbaliser leurs usages et leurs utilisations des technologies, malgré une pratique quotidienne tant dans la sphère scolaire que dans la sphère privée. Pour pallier cette première difficulté, et afin d'encourager une approche pratique de l'usage des TIC(E), nous leur avons demandé une «démonstration" de leur utilisation de l'ordinateur. À ce moment précis, nous n'étions pas totalement extérieure aux observations et aux échanges avec notre interlocuteur. Nous nous sommes inspirée des théories de l'ethnométhodologie et de la sociologie pragmatique pour observer les processus d'usagers en situation (Jouët, 2000). Le but était de garder cet échange simple et concret. Par ailleurs, nous devions systématiquement nous adapter à leur emploi du temps scolaire et médical. Certains soins ne pouvant être anticipés, il nous est arrivé d'être obligée d'interrompre nos entretiens pour que les élèves puissent rencontrer les soignants. On voit ainsi que le planning des patients-élèves est toujours partagé entre l'hôpital et l'école, les soins ayant naturellement priorité sur le temps scolaire. Après la transcription d'entretiens, deux types d'analyse ont été réalisés : une analyse de contenu thématique à partir des verbatims par le logiciel AtlasTi et une analyse interprétative. Ces méthodes d'analyse ont été choisies pour permettre une meilleure compréhension des processus de pensée et des actions des enseignants et des élèves dans leurs et des utilisations des TIC(E), ainsi qu'une mise en évidence des différentes formes d'usage en fonction des problématiques de santé rencontrées.

\section{Les objectifs institutionnels de l'intégration des TIC (E) dans la démarche des soins-études}

\subsection{Point de vue institutionnel}

18 À partir des entretiens réalisés auprès des directeurs, des enseignants dont les référents des TICE, nous avons distingué sept objectifs de la mise en place des TIC(E) pour les patients-élèves :

19 1) Les TIC(E) doivent contribuer à favoriser l'inclusion de l'élève hospitalisé. L'un des objectifs annoncés est clairement de favoriser la sociabilité des patients à travers la communication numérique et de rompre l'isolement. Les TIC(E) doivent développer le lien entre le patient et sa famille et avec les camarades anciens et actuels. Il s'agit donc de favoriser des relations internes et externes qui permettent la transition (passé/présent/ futur) ainsi qu'une transmission d'expériences à travers la messagerie et les blogs.

2) Les TIC(E) permettent à l'équipe enseignante de communiquer avec les parents pour les impliquer dans la scolarité de leurs enfants. Cette communication concerne les absences, 
la transmission des notes ainsi que des bilans sur les acquis scolaires. L'outil favorisé est le cartable numérique commun à l'Éducation nationale.

21 3) Les usages et le degré d'appropriation des TIC(E) facilitent l'accès aux apprentissages de l'autonomie pour les patients-élèves présentant des déficiences visuelles, praxiques ou auditives. Il s'agit d'autodétermination et de but d'accomplissement personnel. L'enjeu est si possible une réintégration progressive dans un milieu ordinaire. Les moyens technologiques sont nombreux: postes informatiques adaptés, souris adaptées, grossissement des caractères, cours vocaux, grands écrans, pc portables, tableaux numériques interactifs et logiciels de reconnaissance vocale. L'objectif est que le patientélève devienne autonome pour une meilleure insertion dans un environnement ordinaire.

4) Les usages et là encore l'appropriation des technologies favorisent l'autonomie et le travail en dehors de la classe à travers la numérisation des cours et la mise à disposition de l'enregistrement audio de certaines leçons («baladodiffusion» notamment pour la pratique des langues étrangères). Il s'agit enfin de renforcer le lien élève/professeur par la messagerie électronique et le cartable électronique. Des salles de travail numérique sont mises à disposition des patients-élèves. Toujours en référence à l'accès à l'autonomie, les TICE doivent également aider à pallier les absences dues aux prises en charge médicales pendant les heures de cours. Des didacticiels sont utilisés afin d'évaluer le travail des élèves qui n'ont pu assister à l'enseignement. Les cours peuvent être rattrapés par une mise à disposition des élèves de la "mémoire " de l'enseignement auquel ils n'ont pas pu assister (sous la forme de cours en ligne, cours vocaux, «cours image » du tableau numérique interactif (TNI), cahier de textes en ligne).

5) Les technologies de l'information et de la communication doivent assurer la continuité pédagogique pour les élèves hospitalisés. Ces outils doivent faciliter la communication avec les professeurs et les groupes de travail de l'établissement afin de garantir une meilleure intégration dans la structure hospitalo-scolaire ainsi qu'un retour dans l'établissement d'origine. Afin de permettre une progression pédagogique cohérente, il s'agit d'échanger les cahiers de texte, de maintenir un contact permanent avec le professeur principal et/ou de la discipline concernée, de coordonner les travaux préexistants engagés dans l'établissement d'origine et de permettre aux patients de travailler les matières non assurées dans l'unité de soins-études.

24 6) Les TIC(E) permettent une «ouverture au monde ». Les documents audio-visuels en langue étrangère, les échanges linguistiques internationaux par visioconférences avec des interlocuteurs de pays étrangers sont fortement encouragés.

7) Enfin, les TIC(E) aident à orienter scolairement et professionnellement les élèves hospitalisés. Elles doivent faciliter l'accès à l'information dans le cadre d'un projet personnel et professionnel réalisable. L'appropriation de logiciels et de compétences numériques est largement souhaitée.

\subsection{Décalage entre les objectifs institutionnels affichés et les perceptions et pratiques des élèves}

Sur sept objectifs institutionnels affichés, on constate que seulement trois objectifs sont repris dans les discours des élèves et transparaissent dans les observations de leurs pratiques que nous avons réalisées. Tout d'abord, l'objectif institutionnel $n^{\circ} 3$ - relatif à l'apprentissage de l'autonomie par les patients-élèves présentant des déficiences 
visuelles, praxiques ou auditives en vue d'une insertion en milieu ordinaire - est l'objectif prédominant dans les discours des élèves, les trois-quarts d'entre eux le mentionnant, même s'ils n'évoquent pas le retour en milieu ordinaire. L'objectif $n^{\circ} 6$ faisant état d'une «ouverture au monde » a été clairement mentionné par les deux-tiers des élèves, par exemple via des visites virtuelles. L'objectif $n^{\circ} 1$ lié à l'inclusion de l'élève hospitalisé et à la sociabilité des patients est manifeste dans près des trois-quarts des discours des élèves et à travers leurs pratiques, s'exprimant surtout à travers le maintien du lien avec la famille, les camarades de l'établissement d'origine et autres. Néanmoins, pour les deux CMPA, on note aussi qu'un tiers des élèves se plaignent de l'isolement numérique.

Quant aux autres objectifs ( $\mathrm{n}^{\circ} 2$ : implication des parents; $\mathrm{n}^{\circ} 4:$ autonomie du travail en dehors de la classe ainsi que renforcement du lien élève/enseignant et remède aux absences $; n^{\circ} 5$ continuité pédagogique (notamment avec l'établissement d'origine) ; $n^{\circ} 7$ : orientation scolaire et professionnelle), ils ne sont pas ou guère présents dans les discours des élèves : moins d'un quart. Les objectifs $\mathrm{n}^{\circ} 4$ et $\mathrm{n}^{\circ} 7$ peuvent se présenter de façon plus ponctuelle ou plus limitée dans le temps; là encore, étant sans doute moins sensibles pour les élèves, ils ne se font pas jour dans les discours. L'objectif $n^{\circ} 2$ n'apparaît pas non plus, ce qui peut s'expliquer par le fait que la transmission des absences, des notes, des bilans s'effectue de façon ponctuelle dans les pratiques. L'objectif $n^{\circ} 5$, qui concerne davantage une volonté clairement affichée par l'équipe enseignante des centres scolaires de l'enquête, est moins perçu, moins d'un quart des élèves l'ayant évoqué.

\section{Rapports entretenus par les professionnels avec les $\operatorname{TIC}(\mathrm{E})$}

\subsection{Entre progressisme, volontarisme et pragmatisme}

Les discours des 4 directeurs d'établissement relativement aux TIC(E) sont progressistes. Ils sont marqués par le souci d'une amélioration des conditions d'apprentissage des élèves. Ces professionnels sont partisans d'évolutions technologiques au sein de leur établissement. Leurs discours sur les usages des TIC(E) sont très positifs par la mise en évidence de la personnalisation des techniques d'apprentissage dans le cadre du parcours de soins-études. En outre, les TIC(E) constituent une stratégie d'affichage pour l'institution :

«on voit tous les bienfaits, on observe des progrès extrêmement importants pour certains, les élèves adorent ça. (...) On a eu les moyens pour équiper toutes les salles, on a un matériel extrêmement performant (...) on l'a mis dans nos plaquettes, les familles arrivent, on leur explique quand elles visitent l'école, il y a des journalistes qui sont venus aussi pour ça » (G, DirectriceAdjointe, 41 ans, ÉREA).

Les 5 référents TICE ont un rapport volontariste aux TICE. Ils ont été avant-gardistes dans la mise en œuvre des TIC(E) dans leur établissement, s'attachant à former leurs collègues. Ils se sont constitués un réseau de professionnels spécialisés dans ces technologies. Ils se tiennent très informés des innovations technologiques dans leur domaine :

31 «je me suis documenté, maintenant, je sais à qui m'adresser, je connais bien les fournisseurs. (...) J'ai été un des premiers ici à m'en occuper (...) on s'est occupé d'installer toutes les salles et puis on s'est formé, on a formé les collègues » (H, 37 ans, Référent TICE et enseignant, ÉREA). 
Quant aux discours des enseignants interviewés, les trois-quarts reflètent des usages pragmatiques à visée pédagogique : «La géométrie, c'est difficile, là, ils sont tout de suite dedans, le dessin est propre » ( $\mathrm{T}$, enseignante, 39 ans, CMPA2).

\subsection{Ambiguïté institutionnelle : entre libre usage et contrôle}

L'utilisation des $\operatorname{TIC}(\mathrm{E})$ de manière intensive pose problème tant à l'institution hospitalière qu'aux établissements scolaires. Les entretiens effectués avec les enseignants et les directeurs révèlent, pour une moitié d'entre eux, une ambiguïté et sont marqués par une hésitation entre libre usage et contrôle de l'usage des TIC(E). Si internet et l'utilisation de l'ordinateur, tout en étant adaptés à l'âge de l'élève, ont été largement encouragés dans le temps scolaire, permettant une ouverture vers l'extérieur comme cela a été mentionné par les trois-quarts des enseignants et directeurs, l'utilisation personnelle en a été limitée. Dans ce contexte, l'intensité de l'usage, par les élèves, dépendait fortement de l'hôpital et du contrôle de l'école :

4 «c'est compliqué cette affaire là, parce qu'ils arrivent à un âge où ils ont besoin de liberté et surtout ici et en même temps, il faut être très vigilant, on ne peut pas les laisser. En même temps, on pourrait se dire qu'ils doivent apprendre à se gérer et gérer le temps, ça fait partie de l'apprentissage » (F, enseignante, 44 ans, CMPA1).

\section{Usages pédagogiques des TIC(E)}

\subsection{Le regard des enseignants}

\subsubsection{Entre usage et non-usage}

35 Nous avons interrogé des enseignants, quasiment autant d'hommes (12) que de femmes (13) qui utilisent ou parfois n'utilisent pas les TIC(E) dans le cadre de leurs pratiques professionnelles. Même si nous étions dans une démarche qualitative et que nos entretiens n'étaient pas représentatifs, nous avons observé des facteurs qui semblaient avoir une influence sur l'implication dans la «techno-pédagogie». Même si ce n'est pas systématique, plus des trois-quarts des plus jeunes professeurs (moins de 35 ans) ont, par leur appétence et leur formation, davantage d'aptitude à utiliser les TIC(E). A contrario, un tiers des enseignants, plus âgés, témoignent d'un usage très limité des TIC(E), parfois même d'un non-usage et plus rarement d'une forme de réticence manifeste :

«Je vais m'y mettre» (G, enseignant, 63 ans, CMPA2); «je prends le prétexte de l'âge, la retraite arrive pour moi et puis ne jamais être dans le même établissement donc pour s'investir làdessus, c'est pas évident, mais j'ai tort : c'est un outil indispensable » (R, enseignante, 61 ans, CMPA1) ; «Je suis allergique à l'informatique » (K, enseignant, 58 ans, ÉREA).

Néanmoins, les deux-tiers des enseignants utilisent au moins deux fois par semaine, voire quotidiennement, les TIC(E) dans leurs pratiques pédagogiques. On observe aussi que les matières enseignées déterminent l'usage ou le non-usage. Dans les matières scientifiques ou les langues étrangères, l'usage s'impose nettement plus que dans le Français qui s'y prête moins selon certains enseignants. Selon un professeur, un didacticiel crée un environnement divertissant et, loin de les décourager, pousse les élèves à faire des exercices, tout en amenant à une redéfinition des pratiques pédagogiques: «ça peut motiver les élèves à voir le cours autrement: ils découvrent que c'est une nouvelle façon 
d'apprendre. C'est simple, plus direct. (...) Pour nous ça change les habitudes de travail » (C, enseignant, 28 ans, CMPA2).

\subsubsection{Adaptations pédagogiques aux déficiences} élèves: "dans le cas d'un handicap lié à un problème psy, c'est difficile de suivre un cours linéaire, ça aide à visualiser. Pour la structuration d'une phrase, là c'est juste un mot-clé » $(\mathrm{Q}$, enseignante, 46 ans, CMP2). L'utilisation des TIC(E) par les enseignants permet également des adaptations qui ont été évoquées par la moitié des enseignants. Un professeur d'éducation physique et sportive nous a expliqué qu'il avait l'habitude d'utiliser des vidéos en cours pour aider les élèves en fauteuil roulant à comprendre les règles et les stratégies des sports en fauteuil. Ces règles sont propres aux membres du groupe en situation de handicap moteur. usages pédagogiques contribuant à la normalisation du parcours scolaire. Ils ont estimé que non seulement celles-ci aident les enfants à surmonter leur handicap, mais qu'elles permettent également aux élèves de suivre un programme scolaire semblable à celui de leurs homologues des écoles ordinaires: "J'utilise le même logiciel que j'utiliserais dans un établissement ordinaire et ils font la même chose que dans une école classique... » ( $\mathrm{Z}$, enseignante, 36 ans, ÉREA).

\subsubsection{Micro-innovations et adaptations physiques des $\operatorname{TIC}(E)$ pour le bien-être des jeunes patients}

Les enseignants réalisent de micro-innovations de façon pragmatique et intuitive pour aider les élèves à surmonter leurs contraintes. Ces micro-innovations peuvent également s'élaborer en collaboration avec les ergothérapeutes. Nos observations nous ont permis de découvrir concrètement certaines adaptations des nouvelles technologies même si les micro-innovations ne se limitent pas aux TIC(E) pour les élèves ayant des déficiences physiques, comme l'adaptation du fauteuil roulant à l'usage de l'ordinateur portable. Ils mettent au point également des outils pour pallier la motricité très limitée, voir inexistante, par exemple pour assurer le contrôle d'une souris par les mouvements du petit doigt, des yeux ou de la langue. Un professeur de technologie a bricolé un système en bois qui permet aux élèves ayant une déficience motrice d'appuyer simultanément sur les trois touches de leur clavier (touches "ctrl + alt + suppr") qui permettent de reprendre le contrôle de la machine.

\subsection{Usages des $\operatorname{TIC}(E)$ par les élèves hospitalisés}

\subsubsection{Usages et compétences : contextes hospitalier et hors hospitalier}

41 En termes d'usage et de compétences (compétences techniques, relationnelles, interactionnelles, informelles) (Fluckiger, 2008), en référence aux recherches déjà réalisées sur les pratiques numériques juvéniles (Mabilon-Bonfils, 2012; Fluckiger, 2009; Metton, 2004), les jeunes élèves hospitalisés avaient des habitudes et des pratiques relativement similaires à celles des jeunes extérieurs au milieu hospitalier. Notre enquête montre que plus des deux-tiers de leurs usages sont en grande partie axés sur la communication, les recherches ludiques et les jeux-vidéos : «c'est surtout pour parler avec 
mes copains et pour jouer surtout avec mon avatar » (C, 14 ans, CMPA1). La socialisation, la communication et les pratiques ludiques priment la dimension informative et formatrice des usages des TIC(E) à des fins éducatives.

\subsubsection{Chez soi et ailleurs}

Par les trois-quarts des élèves hospitalisés que nous avons rencontrés, les usages des TIC (E) sont décrits sous l'angle de l'appropriation, de l'autonomie et de l'évasion qu'elles procurent : " on apprend par nous-mêmes » (I, une élève, 21 ans, CMPA2). Plus d'un tiers a cité les voyages en ligne et les découvertes possibles avec internet, ce qui procure aux élèves un sentiment d'évasion qui ouvre de véritables espaces entre un "chez-soi » et « un ailleurs ». Nous avons pu noter dans leurs discours la prise de conscience d'une plus grande autonomie procurée par l'utilisation des TIC(E), qui sont à leurs yeux un " outil pour se grandir » et un espace indépendant à l'abri du regard des parents : " moi, j'adore regarder les voyages, (...) après y'a des visites de musées et quand on ne peut pas y aller, parce qu'ici on est loin, c'est génial » (S, une élève, 17 ans, CMPA1).

\subsubsection{Auto-publication}

Un quart des élèves ont évoqué leur rédaction d'un journal intime (blog) qui peut rendre visible ou non une partie de leur parcours et des événements vécus. Cette forme d'autoécriture est d'emblée partagée et induit une réflexion sur soi et sur autrui :

"moi,j'essaie d'écrire tous les jours, des fois je relis et je trouve que ce que j'ai écrit, c'est pas mal et y'a une période, j'étais vraiment pas bien, ça m'a vraiment aidé, même si ce que j'écrivais, c'était vraiment déprimant quand je vois ça maintenant » (T, 15 ans, un élève, CMPA2).

On peut imaginer que la dimension réflexive permise par l'auto-publication a d'autant plus de force qu'elle se déroule dans un contexte hospitalier. En ce sens, les usages des TIC(E) alimentent la réflexion identitaire de l'élève-patient qui s'élargit en une réflexion sur son unité biographique (avant la maladie, pendant et dans une projection future avec sa maladie intégrée à son identité et à son histoire).

\section{Formes d'usage et d'utilisation des TIC(E)}

\subsection{En relation avec les types de pathologies des élèves}

Sur la base d'entretiens avec les enseignants, dans le cadre du parcours de soins-études, nous avons pu mettre en évidence les différentes et principales formes d'utilisation des TIC(E) en fonction des types de pathologies des élèves.

Tableau 1 : Formes d'usage et d'utilisation des TIC(E) en relation avec les types de pathologies

\begin{tabular}{|l|l|l|l|l|}
\hline $\begin{array}{l}\text { Types de } \\
\text { pathologies }\end{array}$ & $\begin{array}{l}\text { Utilisation } \\
\text { des TIC(E) }\end{array}$ & Rôles des TIC(E) & Effets prédominants des TIC(E) \\
\hline
\end{tabular}




\begin{tabular}{|c|c|c|c|}
\hline $\begin{array}{l}\text { Cyber-dépendance } \\
\text { (addiction aux } \\
\text { jeux-vidéos et } \\
\text { cyber addiction) }\end{array}$ & $\begin{array}{l}\text { Utilisation } \\
\text { limitée }\end{array}$ & $\begin{array}{l}\text { Usage scolaire } \\
\text { uniquement }\end{array}$ & $\begin{array}{l}\text { Utilité dans des proportions } \\
\text { considérées comme raisonnables } \\
\text { pour éviter l'isolement } \\
\text { numérique des élèves ayant } \\
\text { progressivement perdu leurs } \\
\text { liens sociaux avec les pairs et la } \\
\text { famille }\end{array}$ \\
\hline $\begin{array}{l}\text { Troubles (phobies } \\
\text { scolaire, } \\
\text { agoraphobie et } \\
\text { hyperactivité) }\end{array}$ & $\begin{array}{l}\text { Utilisation } \\
\text { recommandée }\end{array}$ & $\begin{array}{l}\text { Concentration et } \\
\text { désir d'acquérir de } \\
\text { nouvelles } \\
\text { connaissances }\end{array}$ & $\begin{array}{l}\text { Réassurance et développement } \\
\text { d'un contexte familier }\end{array}$ \\
\hline $\begin{array}{l}\text { Handicaps } \\
\text { physiques } \\
\text { (handicaps visuels, } \\
\text { déficiences } \\
\text { motrices) }\end{array}$ & $\begin{array}{l}\text { Utilisation } \\
\text { recommandée }\end{array}$ & $\begin{array}{l}\text { Concentration et } \\
\text { désir d'acquérir de } \\
\text { nouvelles } \\
\text { connaissances }\end{array}$ & $\begin{array}{l}\text { Créativité, autonomie et espaces } \\
\text { d'évasion }\end{array}$ \\
\hline
\end{tabular}

Les pratiques pédagogiques des enseignants se caractérisent par diverses modalités d'adaptation aux déficiences et pathologies. Leurs discours montrent bien que les adaptations sont pensées dans la logique catégorielle de la situation de déficience(s) :

«Les TICE constituent des aide-mémoire pour répondre à des difficultés mnésiques. Elles viennent suppléer l'écriture " (G, enseignant, 43 ans, ÉREA) ; «Le développement de la musique assistée par ordinateur permet de répondre à des difficultés attentionnelles (enregistrements, prises de son) et des situations de handicap moteur (synthétiseurs tactiles, microphones etc...)" (K, enseignante, 29 ans, CMPA2) ; «J'ai trois élèves tétraplégiques : la seule manière d'écrire, il n'y a pas d'autres solutions, c'est le logiciel avec la pastille » (N, enseignante, 36 ans, ÉREA).

Pour des pathologies telles que l'addiction aux jeux vidéo, la cyberdépendance, l'utilisation des TIC(E) est volontairement très limitée et contrôlée. Pour ces dépendances, les établissements se trouvent dans une position délicate et ambiguë car un tiers des élèves que nous avons pu interviewer expliquent souffrir de l'isolement numérique. Les élèves ont progressivement perdu les liens sociaux tissés avec les pairs de l'établissement d'origine: "c'est compliqué pour eux car c'est un mal nécessaire mais en même temps, il faut faire aussi attention à ne pas couper tous les liens mais comme on ne sait pas forcément » ( $\mathrm{Y}$, enseignante, 46 ans, CMPA1).

En revanche, pour des troubles tels que la phobie scolaire, l'agoraphobie et l'hyperactivité, l'utilisation des TIC(E) est clairement recommandée par les enseignants. Pour répondre à ces troubles, l'usage des TIC(E) permet de stimuler la concentration et la volonté d'acquérir de nouvelles connaissances. Par ailleurs, les TIC s'inscrivent dans un contexte familier et quotidien, ce qui constitue un élément rassurant. L'utilisation des TIC (E) est aussi clairement recommandée pour les situations de handicap physique qui sont considérées comme les « moins difficiles » $(\mathrm{R}, 43$ ans, CMPA2) à gérer par des enseignants.

\subsection{Avant et pendant l'hospitalisation}

51 Des discours des élèves, trois principaux usages des TIC(E) se dégagent: personnel, scolaire et relatif à la santé. Ces usages et les utilisations concernent aussi bien l'avant 
hospitalisation - dans le contexte familial ou en milieu scolaire ordinaire - que la période d'hospitalisation.

Tableau 2 : Formes d'usage et d'utilisation des TIC(E) avant et pendant l'hospitalisation

\begin{tabular}{|c|c|c|c|}
\hline Formes d'usages des TIC(E) & $\begin{array}{l}\text { Avant } \\
\text { l'hospitalisation }\end{array}$ & $\begin{array}{l}\text { Pendant } \\
\text { l'hospitalisation }\end{array}$ & $\begin{array}{l}\text { Effets des } \\
\text { TIC(E) }\end{array}$ \\
\hline Usage personnel & $\begin{array}{l}\text { Utilisation } \\
\text { prédominante }\end{array}$ & Utilisation limitée & Bien-être \\
\hline Usage scolaire & Utilisation limitée & $\begin{array}{l}\text { Utilisation } \\
\text { prédominante }\end{array}$ & $\begin{array}{l}\text { Réduction de } \\
\text { la perte des } \\
\text { repères }\end{array}$ \\
\hline $\begin{array}{l}\text { Usage relatif à la santé : } \\
\text { informations sur la recherche } \\
\text { médicale et recherche de } \\
\text { communication avec des pairs } \\
\text { atteints de la même pathologie }\end{array}$ & $\begin{array}{l}\text { Quasi-absence } \\
\text { d'utilisation }\end{array}$ & $\begin{array}{l}\text { Quasi-absence } \\
\text { d'utilisation }\end{array}$ & Effets limités \\
\hline
\end{tabular}

Plus des trois-quarts des élèves font état d'un usage personnel bien plus limité à l'hôpital qu'en milieu familial, contrairement à l'usage scolaire. Le fonctionnement collectif, l'ambiguité du positionnement des institutions entre un libre usage et un contrôle des usages, ainsi que la nature des pathologies sont autant d'éléments explicatifs d'un usage des TIC plus limité dans les centres de soins-études qu'en milieu familial. Le fait que l'usage scolaire soit, pour les deux tiers des élèves, plus important dans les centres que dans les établissements scolaires ordinaires s'explique par le fait que l'accès aux équipements est systématique dans les classes des deux CMPA et de l'ÉREA, contrairement à ce qui avait lieu dans les établissements scolaires ordinaires que les élèves avaient connus : " non, ça n'a rien à voir, c'est beaucoup plus ici parce que de toute façon dans mon école, y'avait trop de monde pour qu'on ait tous un ordinateur » (V, un élève, 9 ans, ÉREA). L'usage relatif à la santé reste ponctuel, aussi bien avant que pendant l'hospitalisation. Il se fait au moment de la recherche de symptômes, de l'annonce diagnostique ou dans le cas de la recherche d'informations sur de nouveaux traitements suivis par les élèves-patients, beaucoup plus rarement pour échanger avec des pairs atteints de la même pathologie: "pour ça, c'est très rare, au début de la maladie, quand le médecin m'a dit ce que j'avais, j'ai regardé » (H, un élève, 11 ans, CMPA2).

\section{Discussion conclusive}

Alors que des chercheurs ont travaillé sur les écarts de pratiques et de compétences constitutifs d'inégalités sociales (Granjon, 2009; Brotcorne \& Valenduc, 2009), sur la recomposition des clivages sociaux chez les filles et les garçons avec l'avancée en âge (Mercklé \& Octobre, 2012), nous avons fait le choix de nous centrer sur les critères " hospitalisation et types de pathologies » et " usages et utilisations des TIC(E) avant et pendant l'hospitalisation » pour les mettre en lien avec les usages des TIC(E). Néanmoins, tant les usages sont complexes, d'autres classifications auraient pu être envisagées en complément des critères retenus pour cette recherche, en mettant la focale sur les 
caractéristiques relatives au milieu social, aux pratiques des parents, au genre, au lieu géographique du domicile, de l'établissement scolaire d'origine, aux pratiques de loisirs et autres.

Malgré un meilleur équipement informatique dans les centres scolaires hospitaliers que dans les établissements d'origine, les compétences démontrées (Fluckiger, 2008) par les élèves hospitalisés ne semblent pas meilleures que celles des jeunes en milieu ordinaire. La réelle différence réside dans les contextes d'usage dans la mesure où les jeunes hospitalisés subissent en particulier des changements d'ordre spatio-temporel, l'espace hospitalier étant « reclus » (Goffman, 1968) à l'écart du monde extérieur. Même si l'on note un certain décalage entre les objectifs institutionnels d'intégration des TIC(E) dans la démarche de soins-études affichés par les enseignants et directions, et la perception des élèves, le contexte spécifique des soins-études stimule la concrétisation des valeurs pédagogiques et sociales à travers les TIC(E), telles que l'inclusion, la sociabilité et l'autonomie.

Les micro-innovations mises en œuvre par les enseignants de l'enquête paraissent proches de ce que Von Hippel (1988) nomme les «innovations ascendantes» ou par l'usage, ou encore de ce que Lévi-Strauss (1962) désigne, au sens noble et méthodique et de façon métaphorique par «bricolage », où pour le bricoleur "la règle du jeu est de toujours s'arranger avec "les moyens du bord", c'est-à-dire un ensemble à chaque instant fini d'outils et matériaux, hétéroclites au surplus ». Elles présentent un caractère « ordinaire » de l'innovation, cette dernière ayant besoin de "passeurs » (Alter, 2000), ce passage est le fait d'une action quotidienne banale réalisée par l'équipe éducative. Ces professionnels appréhendent des formes d'inventivité des pratiques ordinaires (de Certeau, 1990). Avec des micro-innovations et adaptations par les enseignants, les TIC(E) peuvent augmenter le bien-être des élèves, favoriser leur développement social et personnel, et minimiser l'épreuve de l'hospitalisation. Cette recherche s'inscrit dans le prolongement de ce que bon nombre de sociologues des usages ont mis en évidence (Jouët, 2000), à savoir que les individus s'approprient les outils à des fins d'émancipation personnelle, d'accomplissement ou à des fins de sociabilités. En effet, les usages et les utilisations des TIC(E) dans le milieu hospitalier sont décrits, par les élèves-patients, de façon tout à fait positive et se traduisent par des sentiments de créativité, d'évasion et par une démarche réflexive. L'exemple le plus emblématique est sans doute celui de l'autopublication comme forme d'écriture sur soi médiatisée par l'ordinateur et internet. En découlent des effets directs et indirects décrits par les élèves en termes d'estime de soi et de construction de l'identité personnelle et sociale. Cette affirmation corrobore ce que Cardon et Delaunay-Téterel (2006) ont mis en évidence en travaillant sur les blogs, à savoir « la production de soi comme technique relationnelle ».

Par conséquent, outre l'apprentissage et la non-rupture avec l'enseignement qu'elles permettent aux élèves hospitalisés évoluant dans un espace de réclusion (Goffman, 1968) et physiquement coupés donc du monde extérieur, les TIC(E) aident à vivre autrement, sur un mode symbolique, l'espace hospitalier. La double analyse, descriptive et interprétative des usages et des utilisations des TIC(E), dans le contexte tout à fait spécifique des élèves connaissant un parcours de soins-études, nous amène à penser le changement de condition favorisé par l'usage des TIC(E) qui offre aux élèves la possibilité de recréer un environnement d'apprentissage, non prioritaire à l'hôpital, et fait d'eux des acteurs. C'est davantage le contexte d'usage que l'usage lui-même qui contribuerait à une perception positive des TIC(E). En effet, dans le contexte scolaire, ce sont les adaptations 
personnalisées des TIC(E) aux élèves et non l'inverse qui procurent un sentiment positif de leur usage. Alors que dans le cadre de pratiques personnelles et sociales, ce sont les élèves qui personnalisent l'usage des TIC(E) en fonction des outils à leur disposition.

À l'issue de cette enquête, s'ouvrent deux perspectives majeures: la première consisterait, dans une approche longitudinale, à étudier les effets des usages et des utilisations des TIC(E) en contexte hospitalier sur la biographie de l'élève. Il s'agirait alors de prendre davantage en considération la dimension temporelle à moyen et à long terme de ces usages et ces utilisations par les élèves, qui s'ajouterait au contexte d'usage. L'autre perspective viserait à conduire une enquête dans un autre espace "reclus " (Goffman, 1968), tel que le milieu carcéral, auprès de jeunes mineurs pour, là encore, comprendre le contexte d'usage des TIC(E), leur appropriation et leur sens dans un milieu tout à fait singulier.

\section{BIBLIOGRAPHIE}

Akrich, M., \& Méadel, C. (2002). Prendre ses médicaments/prendre la parole : les usages des médicaments par les patients dans les listes de discussion électroniques, Sciences Sociales et Santé, 20(1), 89-116.

Alter, N. (2000). L'innovation ordinaire, Paris : PUF.

Aoudé, P. (2011). Les futurs enseignants du primaire face aux TIC : questions de compétences et de formation. Le cas du tableur. Université René Descartes - Paris V. En ligne https://tel.archivesouvertes.fr/tel-00767440/document (consulté le 17 avril 2015).

Baron, G-L., \& Bruillard, É. (2008). Technologies de l'information et de la communication et indigènes numériques : quelle situation ?. Rubrique de la revue STICEF, 15, En ligne http:// sticef.univ-lemans.fr/num/vol2008/09r-baron/sticef_2008_baron_09.htm (consulté le 17 avril 2015).

Baron, G. L., \& Bruillard, É. (1996). L'informatique et ses usagers dans l'éducation. Paris : PUF.

Bourdon, P., \& Roy, J. (2006). Apprendre, un projet de vie. In P. Bourdon \& J. Roy (Éds.), Enfants malades ou accidentés, Quand l'école va au domicile (pp. 27-41). Paris : Édition Delagrave.

Boutet, M., Rocque, S., Langevin, J. \& Dionne, C. (2003). Les défis de l'intégration sociale. Une perspective écologique. Les Cahiers de l'actif, 294/295, 17-27.

Brotcorne, P., \& Valenduc, G. (2009). Les compétences numériques et les inégalités dans les usages d'internet : Comment réduire ces inégalités ?. Les cahiers du numérique. Fracture numérique et justice sociale, 5(1), 45-68.

Callon, M., \& Latour, B. (1991). La science telle qu'elle se fait. Paris : La Découverte.

Cardon, D. \& Delaunay-Téterel, H. (2006). La production de soi comme technique relationnelle. Un essai de typologie des blogs par leurs publics. Réseaux, 4(138), 15-71.

de Certeau, M. (1990). L'invention du quotidien. 1 Arts de faire. Paris : Gallimard. 
Chambat, P. (1994). Usages des technologies de l'information et de la communication (TIC). TIS, 6 (3), 249-270.

Chaptal, A. (2007). Usages prescrits ou annoncés, usages observés. Réflexion sur les usages scolaires du numérique par les enseignants, 10, 81-106.

Dauphin, F. (2012). Culture et pratiques numériques juvéniles : quels usages pour quelles compétences?. Questions Vives, 7(17), 37-52.

Davis, F. D. (1989). Perceived usefulness, perceived ease of use and user acceptance of information technology. Mis Quaterly, 13, 319 - 339.

Fluckiger, C. (2008). L'école à l'épreuve de la culture numérique des élèves. Revue française de pédagogie, 163, 51-61.

Fluckiger, C. (2009). Internet et ses pratiques juvéniles. Medialog, 69, 42-45.

Fribourg, B. (2007). Dynamiques des réseaux relationnels et trajectoires sociales d'usages, Thèse de Doctorat, Université de Provence Aix Marseille.

Goffman, E. (1968). Asiles. Étude sur la condition sociale des malades mentaux. Paris : les Éditions de minuit.

Granjon, F. (2011). Fracture numérique. Communications, 88, 67-73.

Grosjean, S., \& Bonneville, L. Logiques d'implantation des TIC dans le secteur de la santé. Revue française de gestion, 172, 145-157.

Harry, I., Gagnayre, R., \& d'Ivernois, J-F. (2008). Analyse des échanges écrits entre patients diabétiques sur les forums de discussion : intérêt pour l'éducation thérapeutique du patient. Distances et savoirs, 6, 393-412.

Jouët, J. (2000). Retour critique sur la sociologie des usages, 18(1), 487-521.

Lacroix J-G., Miege, B., Moeglin P., Pajon, P., \& Tremblay, G. (1993). La convergence des télécommunications et de l'audiovisuel : un renouvellement de perspective s'impose ", in Technologies de l'information et société, 5(1), Montréal et Liège, téléchargeable (mars 2007) http://revuetis.mshparisnord.org/article.php3?id_article=21, 81-105

Leblanc, G. (1998). Médias et dispositifs : une approche comparatiste , Cinémas, 9(1), 11-24.

Le Coadic, R. (2006). « L'autonomie, illusion ou projet de société ». Cahiers internationaux de sociologie, 121, 317-340.

Lévi-Strauss, C. La pensée sauvage. Paris : Plon.

Mabilon-Bonfils, B. (2012). Les " pratiques numériques », entre écrit et sociabilité. Adolescence, 79, 217-228.

Mercklé, P., \& Octobre, S. (2012). La stratification sociale des pratiques numériques des adolescents. RESET, 1, 26-52. En ligne journal-reset. index. /RESET/article/view/3

Moeglin, P. (2005). Outils et médias éducatifs. Une approche communicationnelle. Grenoble : Presses de l'Université de Grenoble.

Metton, C. (2004). Les usages d'internet des collégiens. Explorer les mondes sociaux depuis le domicile. Réseaux, 123, 59-84.

Michaud, P-A., \& Colom-Phelipot, P. L'enfant malade et Internet. (2003) In J.P. Dommergues, G. Leverger G. \& D. Rapoport (Éds.). Droit de savoir, Savoir dire, L'enfant malade. Paris : Belin, 301-324. 
Nabarette, H. (2002). L'internet médical et la consommation d'information par les patients. Réseaux, 114, 249-286.

Peeters, H., \& Charlier, P. (1999). Contributions à une théorie du dispositif. Hermès, 25, 15-23. Quintin, J-J. (2014). Relation entre les technologies de l'information et de la communication pour l'éducation (TICE) et la technologie de l'éducation. En ligne http://www.edu-tice.org/approchetheorique (consulté le 17 avril 2015).

Romano, H. (2006). Malade ou accidenté ? D'une réalité bouleversante à une dynamique bouleversée. In P. Bourdon \& J. Roy (Éds.). Enfants malades ou accidentés, Quand l'étude va au domicile. Paris : Éditions Delagrave, 43-72.

Sands, D.J., \& Wehmeyer, M. L. (Eds.). (1996). Self-determination across the life span: independence and choice for individuals with disabilities. Baltimore : Paul H. Brookes Publishers.

Sturtz, C., \& Marquet, P. (2008). La place des technologies de l'information et de la communication dans le contexte hospitalo-scolaire. Actes de la Conférence Internationale éducation, économie et société, Paris. En ligne https://www.canal-u.tv/video/canal_u_medecine/ ipm_2007_la_place_des_tic_dans_le_contexte_hospitalo_scolaire.3446 (consulté le 17 avril 2015). Vehmeyer, M.L., \& Bolding, N. (1999). Self-determination accross living and working environments: a matched samples study of adults with mental retardation, Mental retardation, 37 (5), 353-363.

Voisin, H. (2003). L'école dans l'hôpital : un autre partage des savoirs. In P. Bourdon \& J. Roy. (Éds.). Enfants malades ou accidentés, Quand l'étude va au domicile. Paris: Éditions Delagrave, 182-218.

Von Hippel, É. (1988). The sources of innovation. New York: Oxford University Press.

Haute autorité de santé. (2011). Enjeux et spécificités de la prise en charge des enfants et adolescents en établissements de santé. Charte de l'enfant hospitalisé, article 7. En ligne http://hassante.fr/portail/upload/docs/application/pdf/2012-02/20120213_guide_pec_enfant_ado.pdf (consulté le 17 avril 2015).

Loi $\mathrm{n}^{\circ}$ 2005-102 du 11 février 2005 pour l'égalité des droits et des chances, la participation et la citoyenneté des personnes handicapées En ligne http://www.legifrance.gouv.fr/affichTexte.do? cidTexte=JORFTEXT000000809647 (consulté le 18 mai 2015).

Loi n 2005-102 du 11 février 2005 pour l'égalité des droits et des chances, la participation et la citoyenneté des personnes handicapées. En ligne http://www.legifrance.gouv.fr/affichTexte.do? cidTexte=JORFTEXT000000809647 (consulté le 17 avril 2015).

Loi $\mathrm{n}^{\circ}$ 2013-595 du 8 juillet 2013 d'orientation et de programmation pour la refondation de l'école de la République. En ligne http://www.legifrance.gouv.fr/affichTexte.do? cidTexte=JORFTEXT000027677984 (consulté le 13 mars 2015).

Ministère chargé de la santé. (2013). Hôpital numérique. La politique nationale relative aux systèmes d'information hospitaliers 2012-2017. En ligne http://www.sante.gouv.fr/IMG/pdf/ Hopital_numerique_-_feuille_de_route.pdf (consulté le 17 avril 2015).

Ministère de l'Éducation nationale, de l'enseignement supérieur et de la recherche. (2015). L'École numérique. En ligne http://www.education.gouv.fr/pid29064/ecole-numerique.html (consulté le 15 mai 2015).

Ministère de l'Éducation nationale, de l'enseignement supérieur et de la recherche. L'École numérique. (2015). En ligne http://www.education.gouv.fr/pid29064/ecole-numerique.html (consulté le 7 mai 2015). 


\section{RÉSUMÉS}

L'objectif de cette recherche est de comprendre les usages et les utilisations des TIC(E) à destination d'élèves suivant un parcours de soins-études. Plus qu'à connaître les usages des TIC (E) propres aux élèves hospitalisés, nous nous sommes surtout attachée à comprendre les rapports qu'entretiennent les enseignants et directeurs d'établissement qui accompagnent ces élèves dans leur usage des TIC(E). La méthodologie repose sur une dizaine d'observations et une soixantaine d'entretiens conduits à la fois auprès de professionnels ainsi qu'auprès d'élèves. Les résultats mettent en évidence les objectifs institutionnels de l'intégration des TIC(E) dans la démarche de soins-études. Au-delà de l'analyse des usages pédagogiques des TIC(E) par les enseignants, l'enquête montre comment les TIC(E) favorisent, par de micro-innovations adaptations, le bien-être des jeunes; comment les TIC(E) sont utilisés par les élèves avant et pendant leur hospitalisation, tout en les mettant en rapport avec leurs types de pathologies.

The purpose of this research is to understand the health education student ICT(E) use. More than getting to know the uses of ICT(E) specific to patient-students, we have focused on understanding the relationships between teachers and school leaders who accompany the students in their ICT (E) use. The methodology is based on ten observations and fifty-nine interviews conducted with both professionals as well as students. The results highlight the institutional objectives of the integration of ICT(E) in the care-study approach. Beyond the analysis of the educational uses of ICT(E) by teachers the survey shows how ICT(E), by micro-innovation modifications, encourages the well-being of young people. It shows how ICT(E) is used before and during hospitalization, and how it puts them in relation to their types of pathologies.

\section{INDEX}

Mots-clés : TIC(E), usages, soins-études, élèves, équipes pédagogiques

Keywords : ICT(E), uses, nursing education, students, teaching teams

\section{AUTEUR}

\section{SÉVERINE COLINET}

Maître de Conférences en Sciences de l'Éducation. Université de Cergy-Pontoise. École Mutations Apprentissages (EA-4507) 